\title{
Enquête sur la qualité microbiologique des viandes commercialisées à Conakry, République de Guinée
}

\author{
V. Niamy ${ }^{1}$ S. Keita ${ }^{1}$ B. Guilloteau ${ }^{1}$
}

Mots-clés

Hygiène de la viande - Analyse microbiologique - Contamination biologique - Vente au détail - Industrie de la viande - Norme - Contrôle de qualité - Guinée.

\begin{abstract}
Résumé
Entre 1992 et 1996, des échantillons de viande ont été prélevés sur les marchés, dans les boucheries et supermarchés de Conakry, pour contrôler la qualité microbiologique des produits proposés aux consommateurs. Si l'on se réfère aux normes européennes, 99 p. 100 des échantillons prélevés dans la filière de vente traditionnelle devraient être rejetés pour cause de contamination élevée par la flore aérobie et par les germes anaérobies sulfitoréducteurs. Dans les étalages des supermarchés, seuls 17,4 p. 100 des échantillons des portions unitaires de viande et 4 p. 100 des viandes hachées satisfaisaient aux critères bactériologiques. L'amélioration de cette situation passe obligatoirement par un perfectionnement des conditions d'hygiène d'abattage et de transport des viandes jusqu'au détaillant.
\end{abstract}

\section{INTRODUCTION}

La République de Guinée dispose de bases législatives permettant d'entreprendre une politique de contrôle de l'hygiène alimentaire. Ainsi, le Ministère de l'agriculture et des ressources animales de Guinée a promulgué, le 21 Avril 1991, une ordonnance réglementant l'inspection et l'hygiène des denrées alimentaires d'origine animale. En outre, une loi relative à la protection des consommateurs, au contrôle des denrées, marchandises et services, et à la répression des fraudes commerciales a été promulguée en 1994. Cette loi introduit la notion de responsabilité des opérateurs économiques pour la sécurité des consommateurs en matière d'hygiène des produits alimentaires. Il reste toutefois à préparer une réglementation définissant les normes de conformité des produits, en particulier des critères d'acceptabilité microbiologiques, pour que la loi puisse être mise en application, dans le domaine de la commercialisation des viandes et produits carnés.

De son côté, la Direction nationale de l'élevage, dans le cadre de son Plan de restructuration, s'est dotée des moyens de contrôler la

1. Laboratoire Central de Diagnostic Vétérinaire, BP 559, Conakry, Guinée qualité bactériologique de ces produits en créant, en 1992, une section d'Hygiène alimentaire, au sein du Laboratoire central de diagnostic vétérinaire (LCVD).

C'est dans ce contexte que le Laboratoire a reçu pour mission initiale d'enquêter sur la qualité bactériologique des viandes sur les lieux de vente de la ville de Conakry, en prenant pour référence de contrôle les critères français et européens.

\section{METHODOLOGIE}

\section{Prélèvements}

Entre 1992 et 1996, l'équipe de la section hygiène alimentaire du LCVD a effectué des prélèvements d'échantillons de viandc sur les différents marchés et sur les petites; boucheries traditionnelles des cinq communes de Conakry, ainsi que dans les supermarchés de la même ville qui disposent d'un rayon de boucherie. Les échantillons prélevés, pesant de 200 à 500 g chacun, étaient maintenus au froid durant leur transport jusqu'au laboratoire, puis conservés congelés jusqu'à leur analyse. 
- Prélèvements sur les marchés et petites boucheries traditionnelles :

Ils ont été effectués sur des pièces de viande non parées, présentées en fractions de carcasse, pouvant être classées dans la catégorie des coupes demi-gros. Elles étaient exposées à l'étal du boucher à la température ambiante et fractionnées à la demande.

- Prélèvements dans les boucheries des supermarchés :

Ils ont été réalisés sur des produits classés, selon les critères réglementaires, comme viandes transformées réfrigérées, vendues soit en portion (steak, filet ou bourguignon), soit hachées ou incorporées dans des saucisses. Ces commerces reçoivent la viande de l'abattoir sous forme de demi-carcasses. La viande est ensuite mûrie en chambre froide pendant 5 à 6 jours, avant d'être transformée.

\section{Techniques microbiologiques}

Selon la réglementation française (arrêté du 21 décembre 1979) et européenne, la qualité microbiologique des produits carnés est fonction des taux de contamination par des catégories définies de microorganismes. Les critères sont exprimés en nombre de germes par gramme, comme l'indique le tableau I.

Un échantillon est considéré satisfaisant si le taux de contamination par gramme est inférieur au critère microbiologique maxi- mum défini par la législation, pour chaque catégorie de contaminants. Un échantillon est considéré comme acceptable si le taux de contamination est inférieur à 10 fois la valeur du critère microbiologique maximum. Au-dessus de ce seuil, la denrée est considérée comme non satisfaisante. Pour les salmonelles, le seul critère d'acceptabilité est l'absence totale de ce germe, dans un échantillon de $25 \mathrm{~g}$.

Les méthodes normalisées et les milieux utilisés au LCVD pour la recherche et le dénombrement de ces microorganismes sont les suivantes :

- flore aérobie mésophile à $30{ }^{\circ} \mathrm{C}$ : NF-V08-011; milieu PCA ;

- coliformes fécaux à $44{ }^{\circ} \mathrm{C}: \mathrm{NF}-\mathrm{V} 08-015$; gélose désoxycholate lactose ;

- salmonelles : NF-V08-013 ; le bouilion de Rappaport Vassiliades est utilisé comme milieu d'enrichissement et l'agar de Rambach pour l'isolement ;

- staphylocoques : NF-VO8-014 ; sur milieu de Baid Parker pour le dénombrement ;

- anaérobies sulfito-réducteurs (ASR) et Clostridium perfrengens : en milieu TSN (référence : Diagnostic Pastcur 64724), aprc̀s $24 \mathrm{~h}$ d'incubation à $46^{\circ} \mathrm{C}$.

\section{Tableau I}

Critères microbiologiques relatifs à quelques catégories de viandes et produits carnés

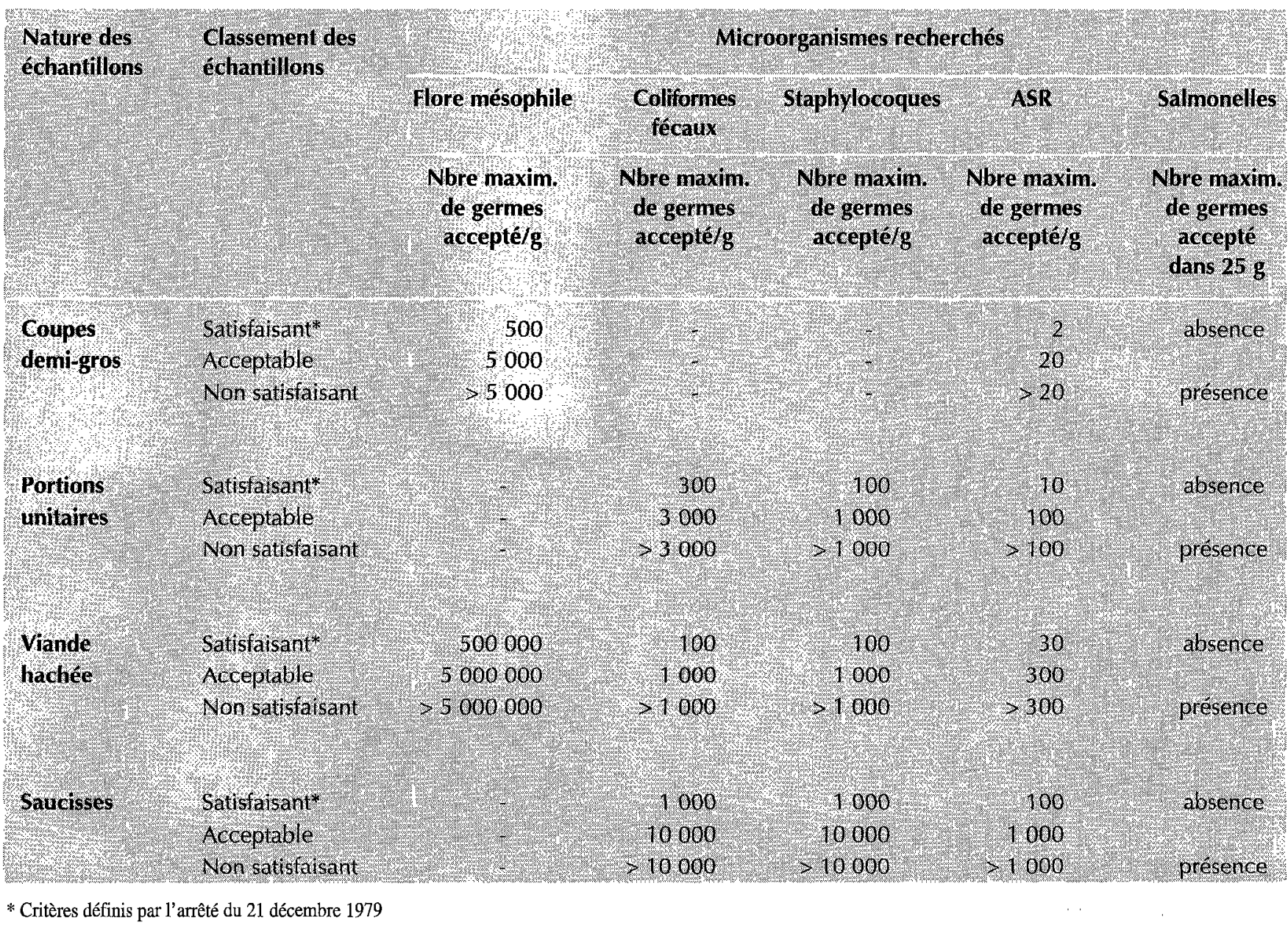




\section{RESULTATS}

Les résultats observés après analyse sont regroupés dans le tableau II.

Sur les pièces de viande vendues dans les petites boucheries traditionnelles, qui peuvent être assimilées à des coupes demi-gros non transformées, aucun échantillon ne pouvait être classé satisfaisant ni acceptable, vis-à-vis de l'ensemble des différents critères microbiologiques applicables à cette catégorie de viande. La quasi-totalité des échantillons ( 99 p. 100) était non satisfaisante en raison du fort niveau de contamination par la flore mésophile. Pour ce qui concerne les germes anaérobies sulfito-réducteurs, 25 p. 100 des échantillons atteignaient ou dépassaient le niveau de rejet à cause de leur présence trop importante. Les taux de contamination moyens de l'ensemble des échantillons par ces deux groupes de germes étaient supérieurs aux taux acceptables. Les salmonelles étaient présentes dans quatre des échantillons.

La recherche des coliformes fécaux, à laquelle ont été ćgalcment soumis ces prélèvements, a révélé un taux moyen élevé de contaminants (21 768 coliformes par gramme).

Pour les viandes transformées, commercialisées dans les supermarchés sous forme de portions unitaires, seuls 17,4 p. 100 des échantillons ont été classés satisfaisants vis-à-vis de l'ensemble des critères microbiologiques. La contamination par les coliformes fécaux était la plus fréquente $(60,8$ p. 100 des échantillons n'étaient pas satisfaisants).

Les coliformes fécaux étaient également un contaminant majeur des viandes hachées (58,3 p. 100 des échantillons n'étaient pas satisfaisants) et des saucisses (51,9 p. 100 des échantillons n'étaient pas satisfaisants). Vis-à-vis de l'ensemble des critères microbiologiques, les viandes hachées et les saucisses n'étaient satisfaisantes que pour respectivement 4 p. 100 et 51,9 p. 100 des prélèvements.

\section{CONCLUSION}

Les viandes non transformées proposées sur les marchés et les petites boucheries traditionnelles étaient particulièrement contaminées et auraient été considérées comme impropres à la consommation dans un contexte d'application des normes européennes, qui imposent que les viandes soient maintenues réfrigérées. Dans le contexte local, les risques encourus par le consommateur sont néanmoins amenuisés, dans la mesure où les habitudes culinaires consistent à ne consommer la viande qu'une fois longuement bouillie. Le risque est par contrc plus important quand ces viandes sont revendues grillées par les vendeurs de brochettes.

\section{Tableau II}

Résultats d'analyse microbiologique regroupés en fonction de la nature des échantillons

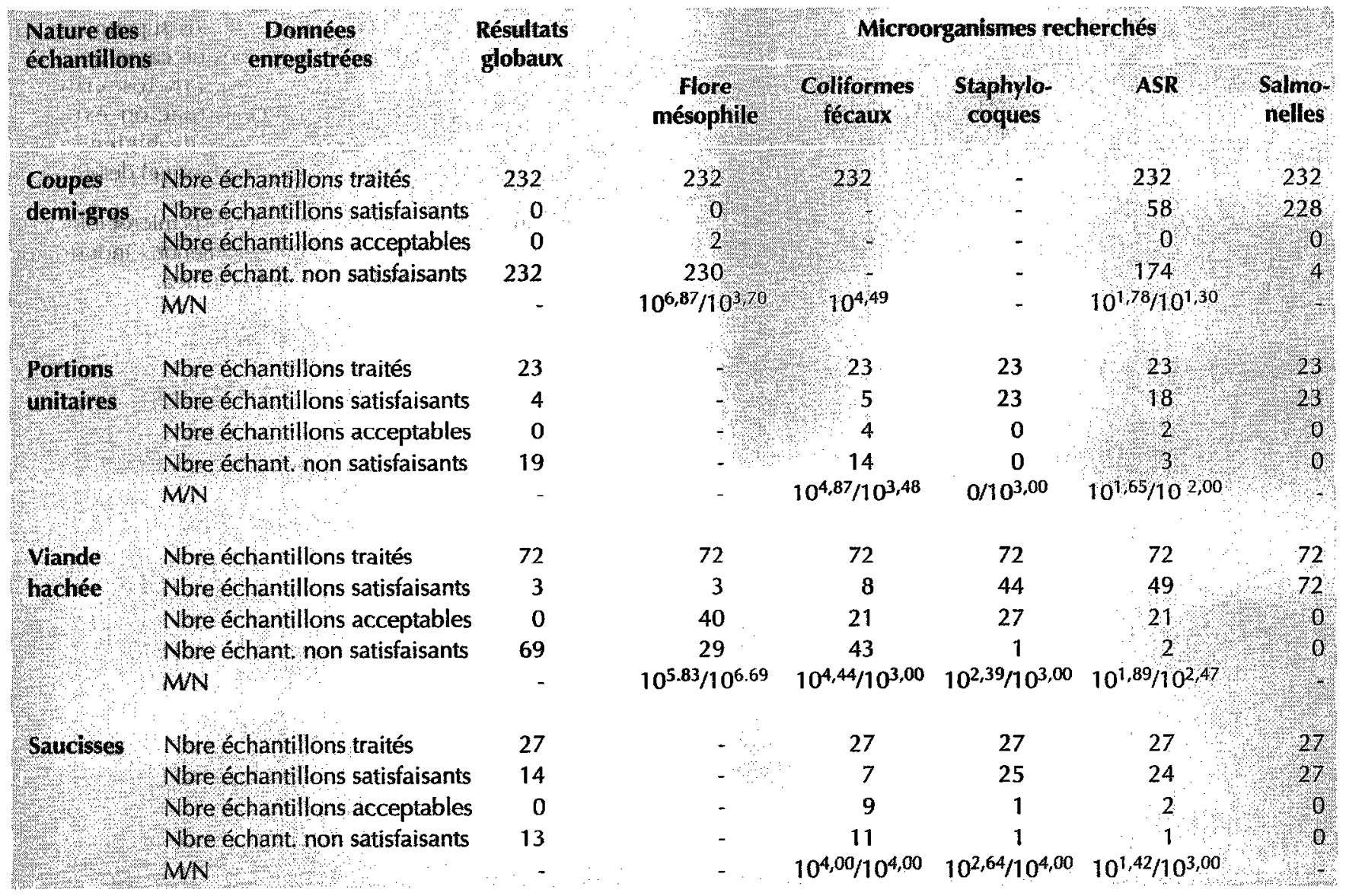

$M$ : contamination moyenne de tous les échantillons de la même catégorie (logarithme du nombre de germes par gramme de viande) $\mathrm{N}$ : contamination maximum d'un échantillon acceptable dans la catégorie (logarithme du nombre de germes par gramme de viande) 
La qualité hygiénique des viandes vendues transformées au niveau des supermarchés est également préoccupante : la majorité des portions unitaires ou des viandes hachées ne satisfont pas aux critères et elles sont destinées à subir un degré moindre de cuisson que les viandes consommées dans la cuisine traditionnelle.

Ces pourcentages élevés de contamination, dans l'une ou dans l'autre filière de commercialisation, démontrent l'insuffisance de la qualité hygiénique de l'ensemble des viandes locales vendues sur la place de Conakry. Ceci est dû à de multiples facteurs dont les plus évidents sont les mauvaises conditions dans lesquelles s'opère encore l'abattage des animaux et $s^{\prime}$ effectue le transport des viandes vers les lieux de vente. Ce n'est qu'une fois ces pro-

\section{Summary}

Niamy V., Keita S., Guilloteau B. Survey on retailed meat microbiological quality in Conakry, Republic of Guinea

Between 1992 and 1996 meat samples were collected for bacteriological quality control from markets, butchers' and supermarkets in Conakry. According to European standards, $99 \%$ of these samples should have been rejecled for high contamination by aerobic flora and sulfito-reducer anaerobic bacteria. In supermarkets only $17.4 \%$ of carved meat serving samples and $4 \%$ of ground meat samples met bacteriological criteria. Better hygiene conditions during slaughtering and transportation to the retailers are crucial to improve the situation.

Key words: Meat hygiene - Microbiological analysis Biological contamination - Retail marketing - Meat industry Standards - Quality control - Guinea. blèmes primordiaux résolus (l'abattoir principal de Conakry fait actuellement l'objet d'un programme de réhabilitation) et après élaboration d'une réglementation normative que des actions de contrôle de qualité au niveau de la commercialisation pourront être entreprises efficacement.

\section{BIBLIOGRAPHIE}

1. BOURGEOIS C.M., LEVEAU J.Y., 1991. Techniques d'analyse et de contrôle dans les industries agro-alimentaires. Cachan, France, Lavoisier, p. 360-368.

Reçu le 27.6.97, accepté le 19.9.97

\section{Resumen}

Niamy V., Keita S., Guilloteau B. Encuesta sobre la calidad microbiológica de las carnes comerciales en Conakry, República de Guinea

Entre 1992 y 1996, se tomaron muestras de carne en los mercados, las carnicerías y los supermercados de Conakry, para controlar la calidad microbiológica de los productos propuestos al consumidor. Si se toman como referencia las normas europeas, $99 \%$ de las muestras examinadas en la filial de la venta tradicional, deberían de ser rechazadas por la alta contaminación con flora aerobia y con gérmenes anaeróbios reductores de sulfitos. En los estantes de los supermercados, solo $17,4 \%$ de las muestras de porciones de carne unitaria y $4 \%$ de las carnes molidas, satisfacían los criterios bacteriológicos. La mejora de esta situación exige un perfeccionamiento de las condiciones de higiene en el matadero y en el transporte de las carnes hasta el detallista.

Palabras clave: Higiene de la carne - Análisis microbiológico Contaminación biológica - Venta al por menor - Industria de la carne - Norma - Control de calidad - Guinea. 